\title{
BMI and age associated variations in the gut microbiome: A pan India, cross sectional study
}

\author{
Kumaresan Nallasamy \\ Tata Chemicals Limited \\ Sucheta Gokhale \\ Tata Chemicals Limited \\ Anirban Bhaduri \\ Tata Chemicals Limited \\ Ashok Kumar Dubey ( $\nabla$ adubey@tatachemicals.com ) \\ Tata Chemicals Limited https://orcid.org/0000-0002-1916-6655
}

\section{Research Article}

Keywords: Gut microbiome, BMI, Age

Posted Date: April 2nd, 2021

DOI: https://doi.org/10.21203/rs.3.rs-370880/v1

License: (9) This work is licensed under a Creative Commons Attribution 4.0 International License.

Read Full License 


\title{
BMI and age associated variations in the gut microbiome: A pan India, cross sectional study
}

${ }^{1}$ Kumaresan Nallasamy, ${ }^{1}$ Sucheta Gokhale, Anirban Bhaduri, Ashok Kumar Dubey*

Affiliations

Innovation Center, Tata Chemicals Ltd, Ambedveth, Pune, Maharashtra, 412111, India

${ }^{1} \mathrm{KN}$ and SG have equally contributed to this work

*Corresponding author

Email: adubey@tatachemicals.com

Orcid ID: 0000-0002-1916-6655

\begin{abstract}
In the current study, we aimed to investigate the association between gut microbiome composition and two physiological factors, BMI and age. We did not observe a significant relationship between occurrence of gut bacteria with BMI or age alone. On the other hand, we observed BMI and age together played an important role in impacting gut microbiota composition. Comparison of the microbiota of normal and obese subjects for the each of 20 s and 50 s group revealed 13 gut bacteria that show significantly different relative abundance in the two groups. We observed that certain organisms show opposite trends within the two age groups. Haemophilus parainfluenzae relative abundance was found to be increased in obese-20s group while reduced in obese-50s group. Relative abundance of organisms such as Mitsuokella jalaludini and Blautia obeum were reduced in obese-20s group while increased in obese-50s group as compared to the normal subjects of respective age group. On the other hand, a reduction in the average relative abundance of both M. jalaludini and B. obeum for obese group as compared to the normal in pan-India only BMI-based group comparison. While studying obesityrelated gut microbiota changes, it is critical to consider multiple factors such as age and geography into the study design.
\end{abstract}

Key words: Gut microbiome, BMI, Age

Gut microbiome composition is influenced by multiple factors such as diet, geography, age, lifestyle, and disease conditions $[1,2]$. Studies have explored the associations between microbiome and various factors for example, age [3], diet [4] and obesity [5]. However, owing to the complex combinatorial effect of multiple factors that shape the overall microbiome, certain observed association vary largely across different populations and a consistent relationship has not been observed yet.

The current study investigates the association between gut microbiome composition and two physiological factors, BMI and age. Subjects were classified into two groups normal and obese based on BMI, and also classified into two age groups viz., 20s and 50s. The geographical location of the subjects was also recorded for further assessment. The LogMPIE [6] data was considered for the analysis. 


\section{BMI associated changes in gut microbiome}

Out of the 993 unique microorganisms identified across the population, 25 reported a significant difference in relative abundance distributions for the pan-India obese and normal population (Figure 1). Since there is a large diversity in dietary habits across India, we further investigated whether the observations were consistent across different zones in India. We observed that many organisms showed a reduction in obesity compared to the normal group. This pattern was observed to be consistent across most of the zones. Organisms such as Victivallis vadensis, Akkermansia muciniphila, Blautia obeum, Coprococcus comes and Eubacterium hadrum were observed to be decreased in the obese population. None of these organisms were observed to be significantly differentially abundant in the groups classified solely based on age. The reduction in the abundance of A. muciniphila, and V. vadensis, that plays an important role in colonizing the gut mucosa layer and modulating the basal metabolism [7], in obese population would mean a thinned mucosa and a leaky gut [8]. Megasphaera micronuciformis, Lactobacillus mисоsae, Dialister invisus were observed to be increased 3 times in the obese population as compared to normal group. Both M. micronuciformis and D. invisus were observed to not differ in relative abundance in the solely age-based groups. L. mucosae was observed to have $\sim 2$ times higher average relative abundance in 50sgroup as compared to 20s-group (p-value 0.055). Interestingly these microorganisms are reported higher in cardiometabolically healthy subjects [9-11]. One possible explanation for the increase in Dialister spp. could be consumption of refined or processed food [12]; however, the increase in $M$. micronuciformis and $L$. mucosae relative abundance in obese population is intriguing.

\section{Age associated changes in gut microbiome}

13 organisms were observed to have significantly different relative abundance distribution between pan-India 20s and 50s groups (Figure 2). From the comparison of microbiome for each zone, we observed that not all organisms obtained as significantly different but the overall trend was similar for most of the zones. The abundance of bacterial species such as Bifidobacterium breve, Actinomyces odontolyticus, Eubacterium ramulus was observed to be reduced in 50s-group. It is known that the Bifidobacteria abundance in the gut reduces with age and the reduction in $B$. breve content is well documented with an occurrence rate ranging from $\sim 5$ to $\sim 19 \%$ in a population of age 50 years and above [13]. However, the interesting observations are the reduced abundance of the oral microbe, A. odontolyticus, which is known to exhibit oral to gut transitioning with their abundance usually more in the aged population $[14,15]$ and the higher abundance of $R$. intestinalis, a known butyrate producer, in the 50s age group compared to 20 s population [16] who are supposedly healthier than 50s. Also interesting is the significance of the increase in the abundance of the not much reported butyrate producer $C$. lavalense [17]. But none of these organisms were observed to have significantly different relative abundance distribution in the solely BMI-based groups.

\section{Combinatorial association of BMI and age with gut microbiome}

Further to our analyses on the BMI and age-based associations of the gut microbiome, we investigated whether BMI and age together playing any role in the gut microbiome composition. We compared the microbiota of normal and obese subjects for the each of 20s and 50s group. We observed 13 organisms that show significantly different relative abundance distribution for normal and obese for the two age groups namely obese-20s and obese-50s (Figure 3). Comparison of these organisms between two age-groups revealed that certain organisms show opposite trends within the two age groups. For instance, Haemophilus parainfluenzae was observed to be 
increased in obese-20s group while reduced in obese-50s group. Interestingly, there is age associated increase in the relative abundance of $H$. parainfluenzae for normal population. Interestingly, for only BMI-based or only age-based groups, no significant difference in the relative abundance distribution was observed. On the other hand, relative abundance of organisms such as Mitsuokella jalaludini and Blautia obeum are reduced in obese20s group while increased in obese-50s group as compared to the normal subjects of respective age group. Similar to the previous observations for both these organisms there is age-associated decrease in the normal population. In case of pan-India only BMI-based group comparison, there is a decrease in the average relative abundance of both $M$. jalaludini and B. obeum for obese group as compared to the normal. These observations suggest a combinatorial association of gut microbes.

In summary, from our exploratory analysis, we observed BMI and age together played an important role in impacting gut microbiota composition. While studying, obesity-related gut microbiota changes, it is imperative to integrate multiple factors such as age and geography into the study design. The current analysis provides a starting point for further multi-factorial analysis of gut microbiome for a larger and diverse population. The present study could pave the way in better understanding of gut bacteria modulation in obese individuals and subsequent microbiota restoration. Further studies need to be conducted to investigate the physiological role of these gut bacteria in obese populations from varied demographic populations.

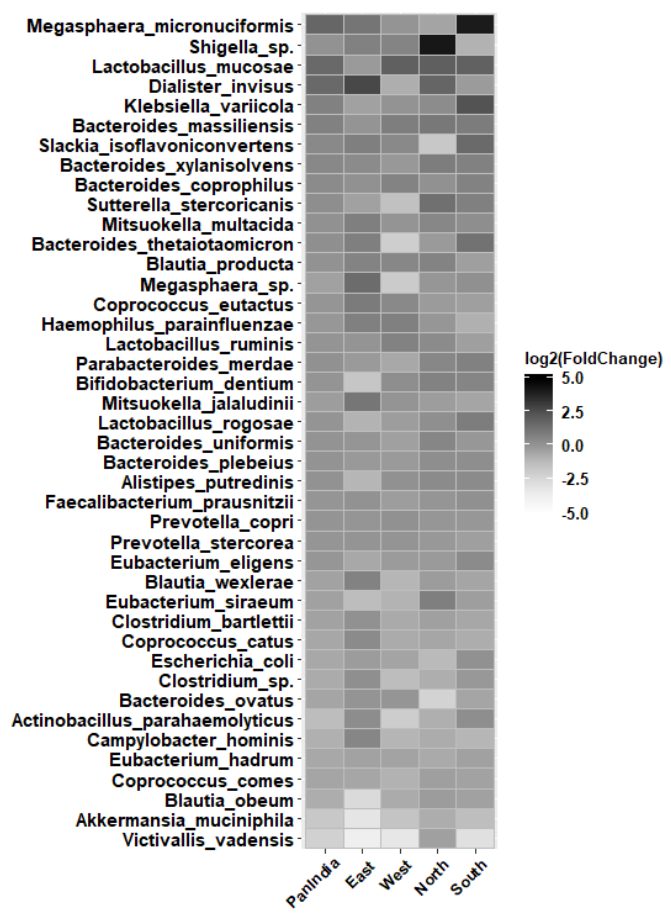

Figure 1: Heat-map of $\log 2$ fold change for organisms with significant difference in relative abundance distribution between BMI based groups for geographical zones in India.

Fold change is the ratio (average relative abundance obese/average relative abundance normal) 


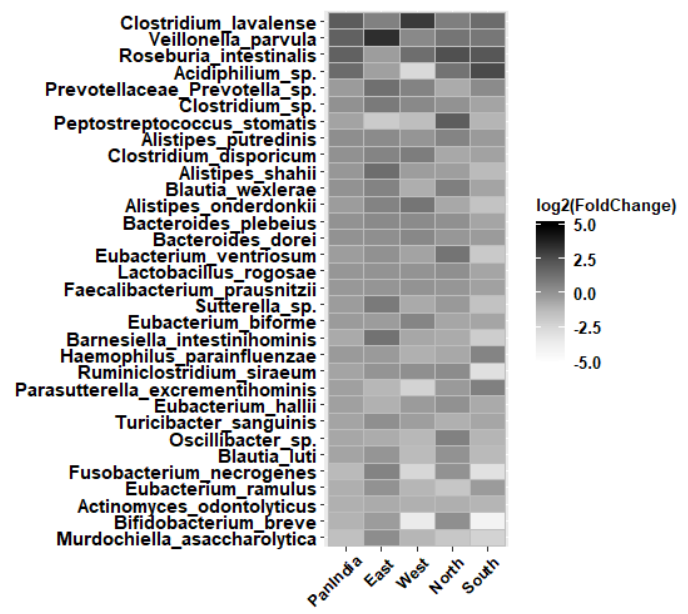

Figure 2: Heat-map of $\log 2$ fold change for organisms with significant difference in relative abundance distribution between age based groups for geographical zones in India.

Fold change is the ratio (average relative abundance old/ average relative abundance young)

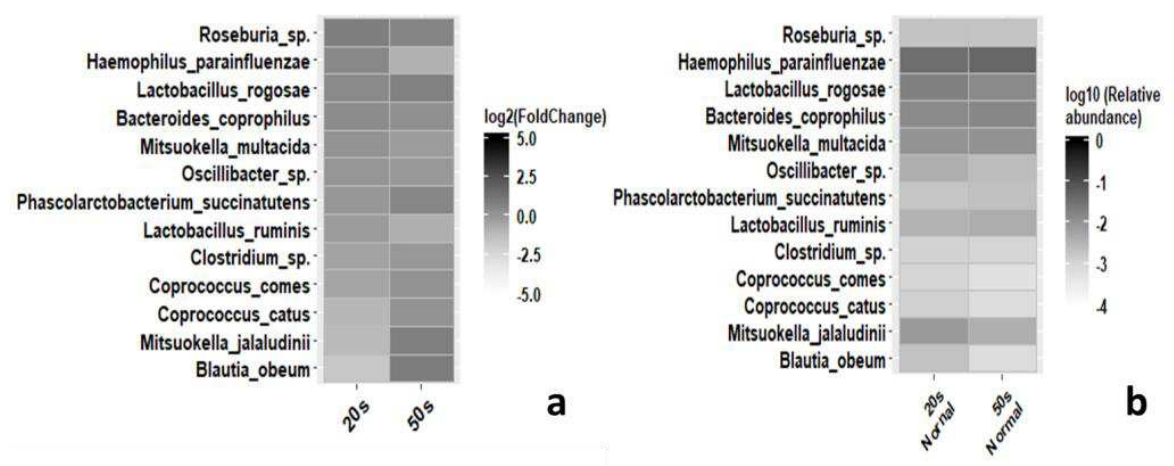

Figure 3: (a) Heat-map of $\log 2$ fold change for organisms with significant difference in relative abundance distribution between BMI based groups for two age groups. Fold change is the ratio (average relative abundance obese/ relative abundance normal) for each age group. (b) Heat-map of log10 average relative abundance for normal subjects of the two age groups.

\section{Methods}

A subject was defined as normal for BMI range of 18.5 to $<25$, and obese for BMI $>=30$. We divided the subjects into two age-based groups. One group with age $<=30$ and the other with age $>=50$. We referred to these groups as 20s-group and 50s-group respectively. Using the information of the city given in [6], four geographical zones were defined. East zone consisted of Guwahati, Patna, and Kolkata. West zone consisted of Ahmedabad, Nagpur, Bhopal, and Mumbai. North zone consisted of Ajmer, Ludhiana, Lucknow, and New Delhi. South zone consisted of Chennai, Mangalore, and Cochin.

Statistical assessment of the microbiome distributions for the subjects was performed using the Mann-Whitney $\mathrm{U}$ test, with a p-value $<0.05$ indicating statistical significance, using in-house written $\mathrm{R}$ codes.

For comparison of relative abundance distribution for each OTU subjects with non-zero abundance were considered. An OTU was not considered for comparison if the number of subjects of each of the two groups 
were $<25$. This translates to the presence of OTU in at least $5 \%$ of total subjects. The ratio of mean relative abundance for each of the comparison was calculated.

\section{Acknowledgements}

The authors would like to acknowledge Faraz Ul Hasan, Madhavi Kaushal, Jyoti Sharma, Bharat Bhushan and Nitish Jha for their assistance in facilitating sample collection. Furthermore, we acknowledge the contribution of Atima Agarwal and Neeraj Chauhan for their inputs in DNA sequencing and Pravin Nilawe for his inputs in primary sequence analysis.

\section{Funding}

This was supported by internal funding from Tata Chemicals Ltd.

\section{Competing interests}

The authors declare no competing interests.

\section{Availability of data and material}

The data related to this study are open and may be accessed from the European Nucleotide Archive (ENA) portal of the European Bioinformatics Institute (https://www.ebi.ac.uk/ena/data/view/PRJEB25642).

\section{Code availability}

Not applicable

\section{Authors' contributions}

A.K.D. designed and conceptualized the study. K.N., S.G., A.B. and A.K.D. looked after the analysis and manuscript preparation. S.G. and A.B. were responsible for the bioinformatics analysis. All authors read and approved the final manuscript.

\section{Ethics approval}

The study protocol was approved by independent ethics committees/institutional review boards (IEC/IRB). The study was conducted in accordance to all the pertinent recommendations by the Indian Council of Medical Research (ICMR) for Biomedical Research on Human Subjects and by the International Conference on Harmonization-Good Clinical Practice (ICH-GCP). The study was registered with the Clinical Trial RegistryIndia (CTRI Number: CTRI/2016/03/007616). The study protocol was approved by independent ethics committees/institutional review boards (IEC/IRB).

\section{Consent to participate}

Prior to initiation of the study, willing volunteers were educated on the study objectives and the sampling procedure, and consent documents were obtained. 


\section{Consent for publication}

Yes

\section{References}

1. Conlon, M.A. and A.R. Bird., The impact of diet and lifestyle on gut microbiota and human health. Nutrients, 2015. 7: p. 17-44.

2. Gaulke, C.A. and T.J. Sharpton, The influence of ethnicity and geography on human gut microbiome composition. Nature medicine, 2018: p. 1495-1496.

3. Yatsunenko, T., F. Rey, and e.a. MJ Manary, Human gut microbiome viewed across age and geography. Nature biotechnology, 2012(486(7402)): p. 222-227.

4. Zhang, N., Z. Ju, and T. Zuo, Time for food: The impact of diet on gut microbiota and human health. Nutrition, 2018. 51.

5. Castaner, O., et al., The gut microbiome profile in obesity: a systematic review. International journal of endocrinology, 2018.

6. Dubey, A.K., et al., LogMPIE, pan-India profiling of the human gut microbiome using $16 S$ rRNA sequencing. Scientific data, 2018. 5: p. 180232.

7. El Kaoutari, A., et al., The abundance and variety of carbohydrate-active enzymes in the human gut microbiota. Nature Reviews Microbiology, 2013. 11: p. 497--504.

8. $\quad$ Earley, H., et al., The abundance of Akkermansia muciniphila and its relationship with sulphated colonic mucins in health and ulcerative colitis. Scientific reports, 2019. 9: p. 1--9.

9. $\quad$ Palleja, A., et al., Roux-en-Y gastric bypass surgery of morbidly obese patients induces swift and persistent changes of the individual gut microbiota. Genome medicine, 2016. 8: p. 67.

10. Ryan, P.M., et al., Lactobacillus mucosae DPC 6426 as a bile-modifying and immunomodulatory microbe. BMC microbiology, 2019. 19: p. 33

11. Farup, P.G., M. Aasbrenn, and J.o.r. Valeur, Separating "good” from "bad" faecal dysbiosis--evidence from two cross-sectional studies. BMC obesity, 2018. 5: p. 30.

12. Davis, S.C., et al., Gut microbiome diversity influenced more by the Westernized dietary regime than the body mass index as assessed using effect size statistic. Microbiology open, 2017. 6: p. e00476.

13. Kato, K., et al., Age-related changes in the composition of gut Bifidobacterium species. Current Microbiology, 2017. 74(8): p. 987-95.

14. Feres, M., et al., The subgingival periodontal microbiota of the aging mouth. Periodontol 2000, 2016. 72(1): p. 30-53.

15. Iwauchi, M., et al., Relationship between oral and gut microbiota in elderly people. Immun Inflamm Dis., 2019.

16. Mäkivuokko, H., et al., The effect of age and non-steroidal anti-inflammatory drugs on human intestinal microbiota composition. Br J Nutr., 2010. 103(2): p. 227-34.

17. Takahashi, K., et al., Reduced abundance of butyrate-producing bacteria species in the fecal microbial community in Crohn's disease. Digestion, 2016. 93: p. 59--65. 


\section{Figures}

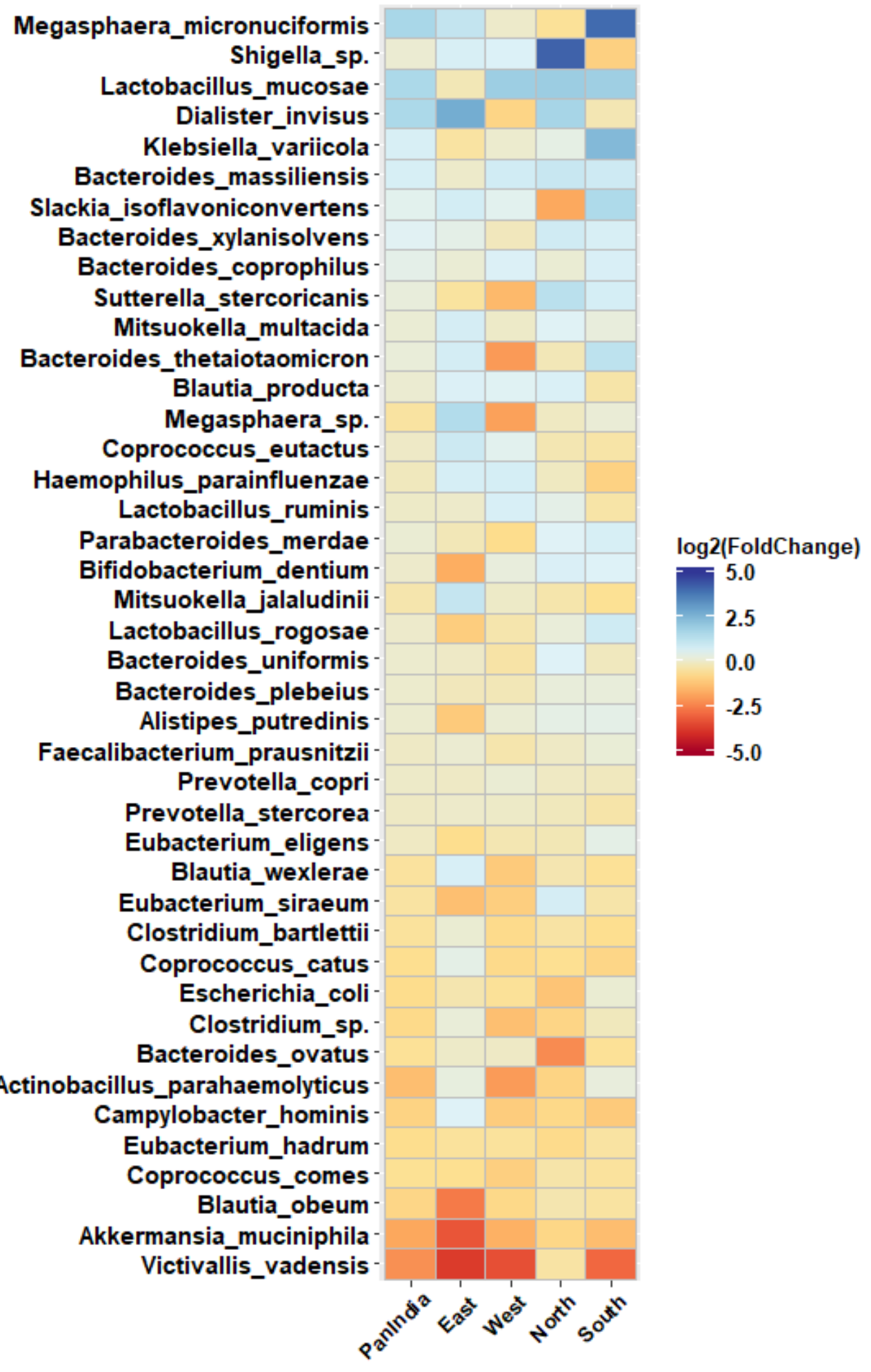

Figure 1

Heat-map of log2 fold change for organisms with significant difference in relative abundance distribution between BMI based groups for geographical zones in India. Fold change is the ratio (average relative abundance obese/average relative abundance normal) 


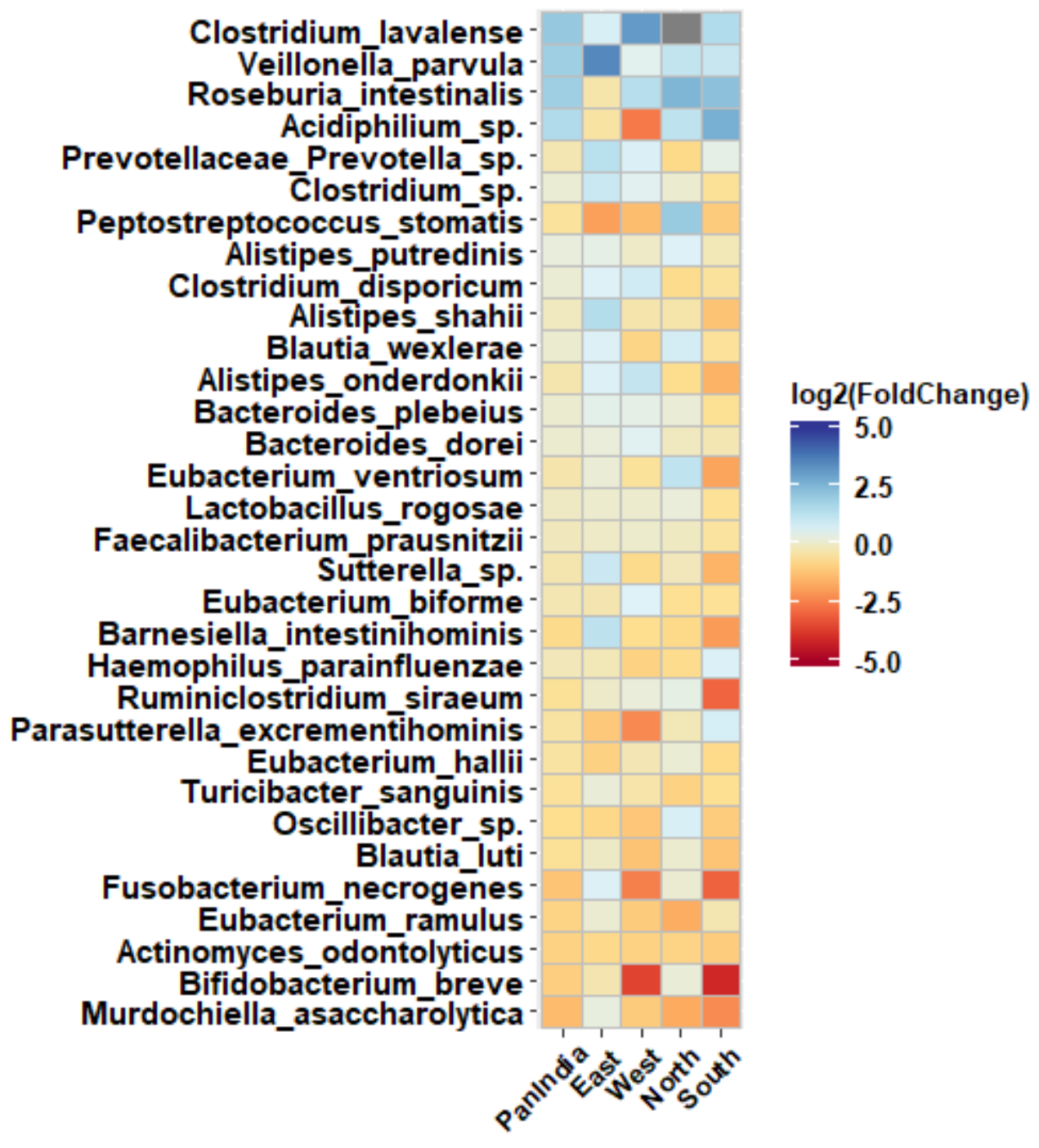

Figure 2

Heat-map of log2 fold change for organisms with significant difference in relative abundance distribution between age based groups for geographical zones in India. Fold change is the ratio (average relative abundance old/ average relative abundance young) 


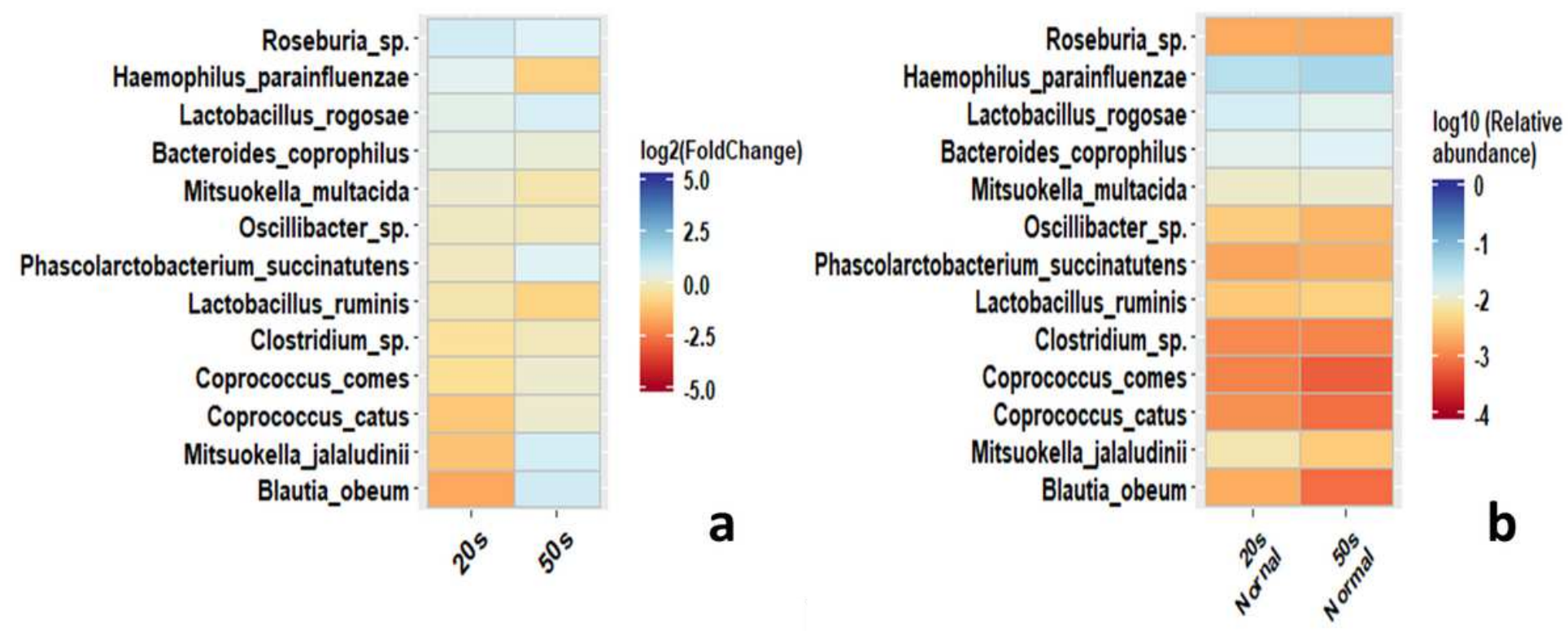

Figure 3

(a) Heat-map of log2 fold change for organisms with significant difference in relative abundance distribution between BMI based groups for two age groups. Fold change is the ratio (average relative abundance obese/ relative abundance normal) for each age group. (b) Heat-map of log10 average relative abundance for normal subjects of the two age groups. 